\title{
BOUNDEDNESS OF VOLTERRA OPERATORS ON SPACES OF ENTIRE FUNCTIONS
}

\author{
Oscar Blasco \\ Universidad de Valencia, Departamento de Análisis Matemático \\ 46100 Burjassot, Valencia, Spain; oblasco@uv.es
}

\begin{abstract}
In this paper we find some necessary and sufficient conditions on an entire function $g$ for the Volterra operator $V_{g}(f)(z)=\int_{0}^{z} f(\xi) g^{\prime}(\xi) d \xi$ to be bounded between different weighted spaces of entire functions $H_{v}^{\infty}(\mathbf{C})$ or Fock-type spaces $\mathcal{F}_{p}^{\phi}(\mathbf{C})$.
\end{abstract}

\section{Introduction}

Let $\Omega$ be the unit disc $\mathbf{D}$ or the complex plane $\mathbf{C}$ and, as usual, denote by $\mathcal{H}(\Omega)$ the space of holomorphic functions in $\Omega$. Given $g \in \mathcal{H}(\Omega)$ the Volterra operator with symbol $g$, to be denoted by $V_{g}$, is defined by

$$
V_{g}(f)(z)=\int_{0}^{z} f(\xi) g^{\prime}(\xi) d \xi, \quad z \in \Omega, f \in \mathcal{H}(\Omega) .
$$

In the case $\Omega=\mathbf{D}$, this operator was first introduced by Pommerenke [20]. He showed that it is bounded on the Hardy space $H^{2}(\mathbf{D})$ if and only if $g \in B M O A$. A bit later the result was extended to $H^{p}(\mathbf{D})$ for any $1 \leq p<\infty$ by Aleman and Siskakis $[1,4]$. In particular, they showed that, for $1 \leq p<\infty$,

$$
\left\|V_{g}(f)\right\|_{H^{p}} \leq C_{p}\|g\|_{B M O A}\|f\|_{H^{p}}, \quad f \in H^{p}(\mathbf{D}),
$$

for a constant $C_{p}>0$ depending only on $p$. The boundedness, compactness and other properties of $V_{g}$ acting on spaces of holomorphic functions defined in the unit disc have been deeply studied (see [5] for weighted Bergman spaces, [6, 15] for weighted spaces of holomorphic functions $H_{v}^{\infty}(\mathbf{D})$ and $[17,19]$ for several other spaces). The reader is also referred to $[2,3]$ for different results concerning the spectra of the Volterra operator in some cases.

In this article we are only concerned with spaces of entire functions. Throughout the paper we write $\mathcal{P}$ for the space of polynomials (with the notation $u_{n}(z)=z^{n}$ ) and $\mathcal{H}_{0}(\mathbf{C})$ for the space of entire functions vanishing at the origin. For each $0<p<\infty$, $0<r<\infty$ and $f \in \mathcal{H}(\mathbf{C})$ we write $M_{\infty}(f, r)=\sup _{|z|=r}|f(z)|$ and $M_{p}(f, r)=$ $\left(\int_{0}^{2 \pi}\left|f\left(r e^{i t}\right)\right|^{p} \frac{d t}{2 \pi}\right)^{1 / p}$.

Given $0<p<\infty$ and a measurable function $\phi:(0, \infty) \rightarrow \mathbf{R}$, we denote by $\mathcal{F}_{p}^{\phi}(\mathbf{C})$ the space of entire functions $f$ such that $\int_{\mathbf{C}}|f(z)|^{p} e^{-p \phi(|z|)} d m(z)<\infty$ and we write

$$
\|f\|_{\mathcal{F}_{p}^{\phi}}=(2 \pi)^{1 / p}\left(\int_{0}^{\infty} M_{p}^{p}(f, r) r e^{-p \phi(r)} d r\right)^{1 / p} .
$$

https://doi.org/10.5186/aasfm.2018.4303

2010 Mathematics Subject Classification: Primary 47G10; Secondary 30H20, 47B07, 47B37, 47B38, 30D15, 30D20, 46E15.

Key words: Volterra operator, weighted spaces, entire function, Fock-type spaces.

The author is partially supported by the Project MTM2014-53009-P(MINECO Spain). 
The classical Fock spaces $\mathcal{F}_{p}(\mathbf{C})$ correspond to $\phi(z)=\frac{|z|^{2}}{2}$.

For the limiting case $\mathcal{F}_{\infty}^{\phi}(\mathbf{C})$ we shall also use the standard notation $H_{v}^{\infty}(\mathbf{C})$ where $v(z)=e^{-\phi(|z|)}$, that is the space of entire functions $f$ such that

$$
\|f\|_{\mathcal{F}_{\infty}^{\phi}}=\|f\|_{v}=\sup _{r \geq 0} e^{-\phi(r)} M_{\infty}(f, r)<\infty .
$$

As usual $H_{v}^{0}(\mathbf{C})$ denotes the subspace of $H_{v}^{\infty}(\mathbf{C})$ of functions such that $\lim _{|z| \rightarrow \infty}$ $v(|z|)|f(z)|=0$.

It is well known that we can change the values of $\phi$ or $v$ in a bounded interval $\left[0, R_{0}\right]$ and even that we can replace $\phi$ for another weight $\varphi$ being continuous and increasing so that $H_{w}^{\infty}(\mathbf{C})=H_{v}^{\infty}(\mathbf{C})$ and $\mathcal{F}_{p}^{\varphi}(\mathbf{C})=\mathcal{F}_{p}^{\phi}(\mathbf{C})$ with equivalent norms. Since we are only interested in spaces containing the polynomials, that is $\mathcal{P} \subset H_{v}^{0}(\mathbf{C})$ or $\mathcal{P} \subset \bigcap_{p>0} \mathcal{F}_{p}^{\phi}(\mathbf{C})$, we shall impose the following assumptions on the weights:

$$
\lim _{r \rightarrow \infty} r^{m} v(r)=0, \quad \forall m \in \mathbf{N},
$$

or

$$
\int_{0}^{\infty} r^{m} e^{-p \phi(r)} d r<\infty, \quad \forall m \in \mathbf{N}, \forall p>0 .
$$

Due to the above considerations we introduce the following definition.

Definition 1.1. We write $\mathcal{W}$ for the class of functions $\phi:[0, \infty) \rightarrow \mathbf{R}$ which are continuous, increasing in $\left[r_{\phi}, \infty\right)$ for some $r_{\phi}>0$ and for each $m \in \mathbf{N}$ satisfy

$$
\sup _{r>0} r^{m} e^{-\phi(r)}<\infty .
$$

Notice that conditions (2), (3) and (4) are in fact equivalent. Examples of weights in $\mathcal{W}$ to have in mind are $\varphi_{\alpha, \beta, \gamma}(r)=\beta r^{\alpha}-\gamma \log r$ for $\alpha, \beta>0$ and $\gamma \geq 0$.

The study of the Volterra operator on certain spaces of entire functions was initiated by Constantin in [11]. She characterized continuity (and compactness) of $V_{g}$ on the classical Fock spaces.

Theorem 1.1. [11, Theorem 1] Let $0<p, q<\infty$ and $0 \neq g \in \mathcal{H}_{0}(\mathbf{C})$.

(i) Case $0<p \leq q$ : $V_{g}$ is bounded from $\mathcal{F}_{p}(\mathbf{C})$ into $\mathcal{F}_{q}(\mathbf{C})$ if and only if $g(z)=$ $a z^{2}+b z$ for some $a, b \in \mathbf{C}$.

(ii) Case $q<p$ : $V_{g}$ is bounded from $\mathcal{F}_{p}(\mathbf{C})$ into $\mathcal{F}_{q}(\mathbf{C})$ if and only if $\frac{1}{q}-\frac{1}{p}<\frac{1}{2}$ and $g(z)=a z$ for some $0 \neq a \in \mathbf{C}$.

Later in collaboration with Peláez [12] the results were extended to a class of Fock-type spaces $\mathcal{F}_{p}^{\phi}(\mathbf{C})$ defined by certain smooth radial weights $\phi$. In [12] certain class $\mathcal{I}$ of twice differentiable and rapidly increasing weights was introduced. This class includes examples such as $\phi(r)=r^{\alpha}$ for $\alpha>2, \phi(r)=e^{\beta r}$ for $\beta>0$ or $\phi(r)=e^{e^{r}}$. For weights in this class they obtained the complete characterization of the symbols $g$ which produce bounded Volterra operators $V_{g}$ acting from $\mathcal{F}_{p}^{\phi}(\mathbf{C})$ into $\mathcal{F}_{q}^{\phi}(\mathbf{C})$ (see [12, Theorem 3]). In particular for $p=q$ they showed that for $0 \neq g \in \mathcal{H}(\mathbf{C})$ and $\phi \in \mathcal{I}$, the Volterra operator $V_{g}$ is bounded on $\mathcal{F}_{p}^{\phi}(\mathbf{C})$ if and only if

$$
\sup _{z \in \mathbf{C}} \frac{\left|g^{\prime}(z)\right|}{1+\phi^{\prime}(|z|)}<\infty
$$

Also they generalized Theorem 1.1 as follows: 
Theorem 1.2. [12, Corollary 25] Let $0<p, q<\infty, 0 \neq g \in \mathcal{H}_{0}(\mathbf{C})$ and $\phi(r)=r^{\alpha}$ with $\alpha>2$.

(i) Case $0<p \leq q$ and $1+(\alpha-2)\left(1-\frac{1}{p}+\frac{1}{q}\right) \geq 0$ : $V_{g}$ is bounded from $\mathcal{F}_{p}^{\phi}(\mathbf{C})$ into $\mathcal{F}_{q}^{\phi}(\mathbf{C})$ if and only if $g$ is a polynomial with $\operatorname{deg}(g) \leq 2+(\alpha-2)\left(1-\frac{1}{p}+\frac{1}{q}\right)$.

(ii) Case $q<p$ : $V_{g}$ is bounded from $\mathcal{F}_{p}^{\phi}(\mathbf{C})$ into $\mathcal{F}_{q}^{\phi}(\mathbf{C})$ if and only if $\frac{1}{q}-\frac{1}{p}<\frac{\alpha-1}{2}$ and $g$ is a polynomial with $\operatorname{deg}(g)<\alpha-2\left(1-\frac{1}{p}+\frac{1}{q}\right)$.

The study for $\mathcal{F}_{\infty}^{\phi}(\mathbf{C})=H_{v}^{\infty}(\mathbf{C})$ was considered by Bonet and Taskinen [9] for certain classes of radial weights $v$. We refer also the interested reader to $[8,11,13]$ for results concerning the spectra of the Volterra operator in this setting. In [9] certain class of weights $\mathcal{J}$ (see conditions appearing in [9, Proposition 3.2]) was introduced. This class includes examples such as $\psi(r)=\beta r^{\alpha}-\gamma \log r-\delta \log (\log (1+r))$, for some $\alpha, \beta>0, \gamma, \delta \in \mathbf{R}, \psi(r)=(\log (1+r))^{1+\epsilon}-\gamma \log r-\delta \log (\log (1+r))$, for some $\epsilon>0, \gamma, \delta \in \mathbf{R}$ or, more generally twice differentiable weights satisfying certain conditions (see [9, Thm 3.6, Thm 3.7]). For such a class, using the notation $\tilde{v}(z)$ for the so-called associate weight of $v$ (see [10]), they obtained (see [9, Theorem 3.4]) that for $0 \neq g \in \mathcal{H}(\mathbf{C}), v(z)=e^{-\phi(|z|)}$ and $w(z)=e^{-\psi(|z|)}$ with $\psi \in \mathcal{J}$, the boundedness of $V_{g}$ from $H_{v}^{\infty}(\mathbf{C})$ into $H_{w}^{\infty}(\mathbf{C})$ is equivalent to the condition

$$
\sup _{z \in \mathbf{C}} \frac{\left|g^{\prime}(z)\right| w(z)}{\psi^{\prime}(|z|) \tilde{v}(z)}<\infty
$$

As a consequence they established the following theorem.

Theorem 1.3. [9, Corollary 3.11] Let $v(r)=e^{-\beta r^{\alpha}}$ for $\beta>0$ and $\alpha \geq 1$ and let $0 \neq g \in \mathcal{H}_{0}(\mathbf{C})$. Then $V_{g}$ is bounded on $H_{v}^{\infty}(\mathbf{C})$ if and only $g$ is a polynomial of $\operatorname{deg}(g) \leq[\alpha]$, where $[a]$ stands for the integer part of $a>0$.

Observe that $V_{g}=\mathcal{I} \mathcal{M}_{g^{\prime}}$ where $\mathcal{M}_{g^{\prime}}(f)=f g^{\prime}$ and $\mathcal{I}(f)(z)=\int_{0}^{z} f(\xi) d \xi$. All the previous results are obtained analyzing the action of $\mathcal{M}_{g^{\prime}}$ and $\mathcal{I}$ on the corresponding spaces independently, and using the equivalent definition of the norm of $f$ in the spaces $H_{v}^{\infty}(\mathbf{C})$ and $\mathcal{F}_{p}^{\phi}(\mathbf{C})$ in terms of the derivative $f^{\prime}$ (see [9, Proposition 3.2]) or Littlewood-Paley formula (see [12, Theorem 10]) respectively.

In this paper we would like to attack the boundedness of the Volterra operator $V_{g}$ (and certain modification of it) directly and not relying on the boundedness of the multiplication or differentiation operators independently. Note that the results in [12] do not apply to $\phi(r)=r^{\alpha}$ for $0<\alpha \leq 2$ and not cover different weights $\phi$ and $\psi$ and the results in [9] cover different weights but only for $p=q=\infty$. We shall present here some necessary and sufficient conditions for the boundedness of $V_{g}$ from $\mathcal{F}_{p}^{\phi}(\mathbf{C})$ into $\mathcal{F}_{q}^{\psi}(\mathbf{C})$ for different parameters $0<p, q \leq \infty$ and different weights $\phi$ and $\psi$ belonging to $\mathcal{W}$, extending and providing some alternative proofs of some results in $[9,11,12]$.

Besides the introduction the paper is divided into four sections. The first section contains some results on the class $\mathcal{W}$ while the second one is devoted to some preliminaries on the Volterra operator $V_{g}$ and its modification $\tilde{V}_{g}(f)(z)=\frac{1}{z} \int_{0}^{z} f(\xi) D g(\xi) d \xi$ where $D g(z)=g(z)+z g^{\prime}(z)$. The main contributions are in the last sections where some necessary and sufficient conditions for the boundedness of $V_{g}$ and $\tilde{V}_{g}$ on weighted spaces of holomorphic functions and Fock-type spaces and their applications are provided. It will be shown (see Corollary 4.6) that the existence of a function $g \neq 0$ such that $V_{g}$ is bounded $\mathcal{F}_{p}^{\phi}(\mathbf{C})$ into $\mathcal{F}_{q}^{\psi}(\mathbf{C})$ implies that $V_{u_{k}}$ is also bounded $\mathcal{F}_{p}^{\phi}(\mathbf{C})$ into $\mathcal{F}_{q}^{\psi}(\mathbf{C})$ for all $k \in \mathbf{N}$ such that $g^{(k)}(0) \neq 0$. This forces some relationship between $p$, 
$q, \phi$ and $\psi$. In particular we will show that there is no entire function $0 \neq g \in \mathcal{H}_{0}(\mathbf{C})$ such that $V_{g}$ maps boundedly $H_{v_{1}}^{\infty}(\mathbf{C})$ into $H_{v_{2}}^{\infty}(\mathbf{C})$ for $v_{i}=e^{-\varphi_{\alpha_{i}, \beta_{i}, \gamma_{i}}}$ for $i=1,2$ whenever $\alpha_{1}>\alpha_{2}$ or $\alpha_{1}=\alpha_{2}$ and $\beta_{1}>\beta_{2}$ or $\alpha_{1}=\alpha_{2}, \beta_{1}=\beta_{2}$ and $\alpha_{1}-\gamma_{2}+\gamma_{1}<1$ (this actually explains the restriction $\alpha \geq 1$ in Theorem 1.3). Moreover once such a function exists it must be a polynomial of degree less or equal than $\alpha_{1}-\gamma_{2}+\gamma_{1}$. In order to provide some sufficient conditions for the boundedness of $V_{g}$ for different weights we shall introduce a function inspired by the so-called distortion function of $\phi$ considered in [12]. For each $0<p<\infty$ and weight $\phi$ the authors considered the function $\psi_{p, \phi}(r)=\frac{\int_{r}^{\infty} s e^{-p \phi(s)} d s}{(1+r) e^{-p \phi(r)}}, r \geq 0$, which was crucial to describe the norm of $f$ in $\mathcal{F}_{p}^{\phi}(\mathbf{C})$ in terms of the derivative $f^{\prime}$. We shall introduce for each pair $(\phi, \psi)$ of weights and $0<p<\infty$ the function

$$
H_{\psi, \phi, p}(r)= \begin{cases}e^{-\phi(r)}\left(\frac{1}{r} \int_{r}^{\infty} e^{-p \psi(s)} d s\right)^{-1 / p}, & 0<p \leq 1 ; \\ e^{-(\phi(r)+(p-1) \psi(r))}\left(\frac{1}{r} \int_{r}^{\infty} e^{-p \psi(s)}, d s\right)^{-1}, & 1<p<\infty\end{cases}
$$

which will play an important role in finding sufficient conditions on the boudedness of $V_{g}$. Namely we shall establish in Theorem 5.7 below that, for $0<p<\infty, \phi, \psi \in \mathcal{W}$ and $g \in \mathcal{H}(\mathbf{C})$, the existence of a constant $A>0$ such that

$$
M_{\infty}(D g, r) \leq A H_{\psi, \phi, p}(r), \quad r>0 .
$$

implies that $\tilde{V}_{g}$ is bounded from $\mathcal{F}_{p}^{\phi}(\mathbf{C})$ into $\mathcal{F}_{p}^{\psi}(\mathbf{C})$. As a consequence one generalizes, at least for $p=q$, the results in [12] to a much wider class of weights.

\section{Preliminaries on weights}

We start by mentioning some classical families of weights. For each $\varepsilon, \alpha, \beta>0$ and $\gamma \in \mathbf{R}$ the consider the weights $\rho_{\varepsilon}$ and $\varphi_{\alpha, \beta, \gamma}$ given by

$$
\rho_{\varepsilon}(r)=(\log (1+r))^{1+\varepsilon}
$$

and

$$
e^{-\varphi_{\alpha, \beta, \gamma}(r)}=\min \left\{(1+r)^{\gamma}, r^{\gamma}\right\} e^{-\beta r^{\alpha}}
$$

that is $\varphi_{\alpha, \beta, \gamma}(r)=\beta r^{\alpha}-\gamma \log (1+r)$ for $\gamma<0$ and $\varphi_{\alpha, \beta, \gamma}(r)=\beta r^{\alpha}-\gamma \log r$ for $\gamma \geq 0$. It is easy to see that $\rho_{\varepsilon}$ and $\varphi_{\alpha, \beta, \gamma}$ belong to $\mathcal{W}$.

The examples $\varphi_{\alpha, \beta, \gamma}$ can be obtained from a single one $\phi(r)=r$ using the following modifications:

$$
\begin{aligned}
\phi_{\beta}(r) & =\phi(\beta r), \quad \beta>0, \\
\phi^{(\alpha)}(r) & =\phi\left(r^{\alpha}\right), \quad \alpha>0, \\
e^{-\phi_{(\gamma)}(r)} & =\min \left\{(1+r)^{\gamma}, r^{\gamma}\right\} e^{-\phi(r)}, \quad \gamma \in \mathbf{R} .
\end{aligned}
$$

It is elementary to see that if $\phi$ belongs to $\mathcal{W}$ then $\phi_{\beta}, \phi^{(\alpha)}$ and $\phi_{(\gamma)}$ also belong to $\mathcal{W}$.

Definition 2.1. Let $0<p<\infty$ and $\phi$ such that $\int_{r}^{\infty} e^{-p \phi(s)} d s<\infty$ for $r>0$. We define, for $r>0$,

$$
\Phi_{p}(r)=-\frac{1}{p} \log \left(\frac{1}{r} \int_{r}^{\infty} e^{-p \phi(s)} d s\right),
$$

or, equivalently $e^{-p \Phi_{p}(r)}=\frac{1}{r} \int_{r}^{\infty} e^{-p \phi(s)} d s$.

Lemma 2.1. Let $0<p<\infty$ and $\phi \in \mathcal{W}$. Then

(i) $\Phi_{p} \in \mathcal{W}$, 
(ii) if $\phi \in C^{1}(0, \infty)$ and convex, then

$$
\sup _{r \geq 0} e^{\phi(r)-\Phi_{p}(r)}<\infty
$$

(iii) if $\phi(r)=\varphi_{\alpha, \beta, \gamma}$ for some $\alpha, \beta>0$ and $\gamma \in \mathbf{R}$, then

$$
\sup _{r>0} r^{\frac{\alpha}{p}} e^{\phi(r)-\Phi_{p}(r)}<\infty .
$$

Proof. (i) Clearly $e^{-\Phi_{p}(r)}$ is decreasing and $\Phi_{p}(r)$ is increasing. Now for each $m \in \mathbf{N}$ with $m p>1$ we have that

$$
r^{p m} e^{-p \Phi_{p}(r)}=r^{p m-1} \int_{r}^{\infty} e^{-p \phi(s)} d s \leq \int_{0}^{\infty} s^{p m-1} e^{-p \phi(s)} d s<\infty .
$$

This shows that $\Phi_{p} \in \mathcal{W}$.

(ii) Note that $r \phi^{\prime}(r) \geq \phi^{\prime}(1)=A$ for $r \geq 1$. Hence for $r \geq 1$

$$
e^{-p \Phi_{p}(r)}=\frac{1}{r} \int_{r}^{\infty} e^{-p \phi(t)} d t \leq \frac{1}{A} \int_{r}^{\infty} \phi^{\prime}(t) e^{-p \phi(t)} d t=\frac{1}{p A} e^{-p \phi(r)} .
$$

Since $\sup _{0<r<1} e^{\phi(r)-\Phi_{p}(r)}<\infty$ this gives $(12)$.

(iii) We claim that for any $a \in \mathbf{R}$ there exists $C_{a}>0$ so that

$$
\int_{r}^{\infty} t^{a} e^{-t} d t \leq C_{a} r^{a} e^{-r}, \quad r>0 .
$$

Of course the result holds true for $a \leq 0$ with $C_{a}=1$. The case $a \in \mathbf{N}$ follows by induction and integration by parts. Now for $a>0$ write $a=\lambda k_{0}+(1-\lambda) k_{1}$ with $0 \leq \lambda \leq 1$ and $k_{0}, k_{1} \in \mathbf{N} \cup\{0\}$, apply Hölder's inequality and the previous case to get (14). To show (13) we consider the cases $\gamma \geq 0$ and $\gamma<0$ separately.

Case $\gamma \geq 0$ : From (14) we have

$$
\begin{aligned}
e^{-\Phi_{p}(r)} & =\left(\frac{1}{r} \int_{r}^{\infty} t^{p \gamma} e^{-p \beta t^{\alpha}} d t\right)^{1 / p}=C\left(\frac{1}{r} \int_{p \beta r^{\alpha}}^{\infty} s^{\frac{p \gamma+1}{\alpha}-1} e^{-s} d t\right)^{1 / p} \\
& \leq C^{\prime} r^{\gamma-\frac{\alpha}{p}} e^{-\beta r^{\alpha}}=C^{\prime} r^{-\frac{\alpha}{p}} e^{-\phi(r)}
\end{aligned}
$$

Case $\gamma<0$ : Arguing as above,

$$
\begin{aligned}
e^{-\Phi_{p}(r)} & =\left(\frac{1}{r} \int_{r}^{\infty}(1+t)^{p \gamma} e^{-p \beta t^{\alpha}} d t\right)^{1 / p} \leq C\left(\frac{(1+r)^{p \gamma}}{r} \int_{p \beta r^{\alpha}}^{\infty} s^{\frac{1}{\alpha}-1} e^{-s} d t\right)^{1 / p} \\
& \leq C(1+r)^{\gamma} r^{-\alpha / p} e^{-\beta r^{\alpha}} \leq r^{-\frac{\alpha}{p}} e^{-\phi(r)} .
\end{aligned}
$$

The proof is complete.

Let us now consider a subclass of differentiable weights wide enough to include most of the classical weights.

Definition 2.2. Let us denote $\mathcal{W}_{0}$ the collection of continuous functions $\phi:[0, \infty)$ $\rightarrow \mathbf{R}$ such that $\phi \in C^{1}\left(\left[r_{\phi}, \infty\right)\right)$ for some $r_{\phi} \geq 0$ and

$$
\lim _{r \rightarrow \infty} r \phi^{\prime}(r)=\infty \text {. }
$$

Note that the classical examples $\varphi_{\alpha, \beta, \gamma}$ and $\rho_{\varepsilon}$ belong to $\mathcal{W}_{0}$ for any $\epsilon, \alpha, \beta>0$ and $\gamma \in \mathbf{R}$.

Lemma 2.2. $\mathcal{W}_{0} \subset \mathcal{W}$. 
Proof. Let $\phi \in \mathcal{W}_{0}$. Then $\phi^{\prime}(r)>0$ in some interval $(R, \infty)$ and for each $m \in \mathbf{N}$, L'Hospital's rule gives $\lim _{r \rightarrow \infty} \frac{\phi(r)-m \log r}{m \log r}=\infty$. In particular $\lim _{r \rightarrow \infty}(\phi(r)-$ $m \log r)=\infty$. Hence (2) holds and then $\phi \in \mathcal{W}$.

Proposition 2.3. Let $0<p<\infty$ and let $\phi$ be differentiable with $\phi^{\prime}(r)>0$ for $r>0$. Then

$$
\phi \in \mathcal{W}_{0} \Longleftrightarrow \Phi_{p} \in \mathcal{W}_{0} \Longleftrightarrow \lim _{r \rightarrow \infty} e^{\phi(r)-\Phi_{p}(r)}=0
$$

Proof. Differentiating in the formula $e^{-p \Phi_{p}(r)}=\frac{1}{r} \int_{r}^{\infty} e^{-p \phi(s)} d s$ one has that $\operatorname{pr} \Phi_{p}^{\prime}(r)=e^{p\left(\Phi_{p}(r)-\phi(r)\right)}+1$. Now use L'Hospital's rule to obtain

$$
\lim _{r \rightarrow \infty} p r \Phi_{p}^{\prime}(r)=\lim _{r \rightarrow \infty} \frac{r e^{-p \phi(r)}}{\int_{r}^{\infty} e^{-p \phi(s)} d s}+1=p \lim _{r \rightarrow \infty} r \phi^{\prime}(r) .
$$

Thus both equivalences are shown.

Let us give a notation to the sequence of the norms of $u_{k}$ in the space $\mathcal{F}_{p}^{\phi}$ for any weight $\phi \in \mathcal{W}$ and $0<p \leq \infty$.

Definition 2.3. Let $0<p \leq \infty, \phi \in \mathcal{W}$ and $k \in \mathbf{N} \cup\{0\}$. We define

$$
\begin{aligned}
C_{k}(\phi, p) & =\left(\int_{0}^{\infty} r^{p k+1} e^{-p \phi(r)} d r\right)^{1 / p}=(2 \pi)^{-1 / p}\left\|u_{k}\right\|_{\mathcal{F}_{p}^{\phi}}, \\
C_{k}(\phi, \infty) & =\sup _{0<r<\infty} r^{k} e^{-\phi(r)}=\left\|u_{k}\right\|_{\mathcal{F}_{\infty}^{\phi}} .
\end{aligned}
$$

Next result is immediate and left to the reader.

Example 2.1. Let $\alpha, \beta, p>0, \gamma \geq 0$ and $\phi=\varphi_{\alpha, \beta, \gamma}$. Then

$$
C_{k}(\phi, \infty)=(\alpha \beta)^{-\frac{k+\gamma}{\alpha}}(k+\gamma)^{\frac{k+\gamma}{\alpha}} e^{-\frac{k+\gamma}{\alpha}}
$$

and

$$
C_{k}^{p}(\phi, p)=\frac{(p \beta)^{-\frac{p k+2+p \gamma}{\alpha}}}{\alpha} \Gamma\left(\frac{p k+2+p \gamma}{\alpha}\right) .
$$

Remark 2.1. For $0<p, p_{1}, p_{2}<\infty, k_{1}, k_{2}, k \in \mathbf{N} \cup\{0\}$ and $\phi, \psi \in \mathcal{W}$ we have

$$
\begin{aligned}
C_{k_{1}+k_{2}}(\phi+\psi, p) & \leq \min \left\{C_{k_{1}}(\phi, p) C_{k_{2}}(\psi, \infty), C_{k_{2}}(\phi, p) C_{k_{1}}(\psi, \infty)\right\}, \\
C_{k}\left(\phi, p_{3}\right) & \leq C_{k}\left(\phi, p_{1}\right) C_{k}\left(\phi, p_{2}\right), \quad \frac{1}{p_{3}}=\frac{1}{p_{1}}+\frac{1}{p_{2}}, \\
C_{k}\left(\phi, p_{2}\right) & \leq C_{k}\left(\phi, p_{1}\right)^{p_{1} / p_{2}} C_{k}(\phi, \infty)^{1-p_{1} / p_{2}}, \quad p_{1}<p_{2} .
\end{aligned}
$$

Lemma 2.4. Let $\phi \in \mathcal{W}$ and $0<p \leq \infty$. Then the sequences $\left(\left(C_{0}^{-1}(\phi, p) C_{k}(\phi, p)\right)^{1 / k}\right)_{k}$ and $\left(C_{k+1}(\phi, p) / C_{k}(\phi, p)\right)_{k}$ are increasing with

$$
\lim _{k} \frac{C_{k+1}(\phi, p)}{C_{k}(\phi, p)}=\lim _{k \rightarrow \infty} C_{k}^{1 / k}(\phi, p)=\infty .
$$

Proof. Case $p=\infty$ : Since $e^{-\phi(r) / k} \leq e^{-\phi(r) /(k+1)}$ for all $r>0$ and $k \in \mathbf{N}$ then obviously $\left(C_{k}(\phi, \infty)^{1 / k}\right)_{k}$ is increasing. Let us show that $\left(\frac{C_{k+1}(\phi, \infty)}{C_{k}(\phi, \infty)}\right)_{k}$ is also increasing. Since $k=\frac{1}{2}(k-1)+\frac{1}{2}(k+1)$, we have that

$$
C_{k}(\phi, \infty)=\sup _{r>0} r^{\frac{(k-1)}{2}} e^{-\frac{\phi(r)}{2}} r^{\frac{(k+1)}{2}} e^{-\frac{\phi(r)}{2}} \leq C_{k-1}(\phi, \infty)^{1 / 2} C_{k+1}(\phi, \infty)^{1 / 2}
$$


and then $C_{k+1}(\phi, \infty) / C_{k}(\phi, \infty)$ is increasing. Finally, using now that $C_{k}(\phi, \infty)^{1 / k} \leq$ $C_{k+1}(\phi, \infty)^{1 /(k+1)}$ we have $\frac{C_{k+1}(\phi, \infty)}{C_{k}(\phi, \infty)} \geq C_{k+1}(\phi, \infty)^{1 /(k+1)}$. Hence $\lim _{k \rightarrow \infty} \frac{C_{k+1}(\phi, \infty)}{C_{k}(\phi, \infty)}=$ $\lim _{k \rightarrow \infty} C_{k}(\phi, \infty)^{1 / k}=\infty$.

Case $0<p<\infty$ : Applying Cauchy-Schwarz we have

$$
\left(\int_{0}^{\infty} r^{p k+p+1} e^{-p \phi(r)} d r\right)^{2} \leq\left(\int_{0}^{\infty} r^{p k+2 p+1} e^{-p \phi(r)} d r\right)\left(\int_{0}^{\infty} r^{p k+1} e^{-p \phi(r)} d r\right) .
$$

This shows that $C_{k+1}(\phi, p)^{2} \leq C_{k+2}(\phi, p) C_{k}(\phi, p)$. Thus $C_{k+1}(\phi, p) / C_{k}(\phi, p)$ is increasing. Now consider the measure $d \mu_{p}(r)=C_{0}(\phi, p)^{-p} r e^{-p \phi(r)} d r$ defined in $\mathbf{R}^{+}$. Of course, $\mu_{p}\left(\mathbf{R}^{+}\right)=1$ and $\left(C_{0}(\phi, p)^{-1} C_{k}(\phi, p)\right)^{1 / k}=\left\|u_{1}\right\|_{L^{p k}\left(\mathbf{R}^{+}, d \mu_{p}\right)}$ where $u_{1}(r)=r$. This gives $\left(C_{0}(\phi, p)^{-1} C_{k_{1}}(\phi, p)\right)^{1 / k_{1}} \leq\left(C_{0}(\phi, p)^{-1} C_{k_{2}}(\phi, p)\right)^{1 / k_{2}}$ whenever $k_{1} \leq k_{2}$. In particular $\left(C_{0}(\phi, p)^{-1} C_{k}(\phi, p)\right)^{1 / k}$ is increasing. Now taking into account that

$$
\frac{C_{k+1}(\phi, p)}{C_{k}(\phi, p)} \geq\left(C_{0}^{-1}(\phi, p) C_{k+1}(\phi, p)\right)^{1 /(k+1)}=\left\|u_{1}\right\|_{L^{p(k+1)}\left(\mu_{p}\right)}
$$

we conclude that $\lim _{k \rightarrow \infty} \frac{C_{k+1}}{C_{k}} \geq \lim _{k \rightarrow \infty}\left\|u_{1}\right\|_{L^{p(k+1)}\left(\mu_{p}\right)}=\left\|u_{1}\right\|_{L^{\infty}\left(\mu_{p}\right)}=\infty$. The proof is complete.

Lemma 2.5. Let $\phi \in \mathcal{W}$ and $0<p \leq \infty$. Then

$$
C_{k}(\phi, p) \leq C_{k_{1}}(\phi, p)^{\frac{k_{2}-k}{k_{2}-k_{1}}} C_{k_{2}}(\phi, p)^{\frac{k-k_{1}}{k_{2}-k_{1}}}, \quad k_{1} \leq k \leq k_{2} .
$$

In particular $C_{k}^{2}(\phi, p) \leq C_{2 k}(\phi, p) C_{0}(\phi, p)$ for all $k \in \mathbf{N}$.

Proof. Let us denote $\mathrm{M}_{k}=C_{k}(\phi, \infty)$ and $\mathrm{C}_{k}=C_{k}(\phi, p)$ for $0<p<\infty$. We start with the case $p=\infty$. For each $k, k_{1}, k_{2} \in \mathbf{N}$ such that $\frac{1}{k}=\frac{\theta}{k_{1}}+\frac{1-\theta}{k_{2}}$, we obviously have $\mathrm{M}_{k}^{1 / k} \leq \mathrm{M}_{k_{1}}^{\theta / k_{1}} \mathrm{M}_{k_{2}}^{(1-\theta) / k_{2}}$. Hence for each $k_{1} \leq k \leq k_{2}$, choosing $\theta=\frac{k_{1}}{k} \frac{k_{2}-k}{k_{2}-k_{1}}$ one obtains $\mathrm{M}_{k} \leq \mathrm{M}_{k_{1}}^{\frac{k_{2}-k}{k_{2}-k_{1}}} \mathrm{M}_{k_{2}}^{\frac{k-k_{1}}{k_{2}-k_{1}}}$.

For $0<p<\infty$, arguing as in the previous lemma we can write for $k_{1} \leq k \leq k_{2}$ and $\frac{1}{p k}=\frac{\theta}{p k_{1}}+\frac{1-\theta}{p k_{2}}$ that

$$
\left\|u_{1}\right\|_{L^{p k}\left(\mathbf{R}^{+}, d \mu_{p}\right)} \leq\left\|u_{1}\right\|_{L^{p k_{1}}\left(\mathbf{R}^{+}, d \mu_{p}\right)}^{\theta}\left\|u_{1}\right\|_{L^{p k_{2}\left(\mathbf{R}^{+}, d \mu_{p}\right)}}^{1-\theta} .
$$

Now (23) follows since $\theta=\frac{k_{1}}{k} \frac{k_{2}-k}{k_{2}-k_{1}}$ and $1-\theta=\frac{k_{2}}{k} \frac{k-k_{1}}{k_{2}-k_{1}}$.

Finally selecting $k_{1}=0$ and $k_{2}=2 k$ one gets $\mathrm{M}_{k}^{2} \leq \mathrm{M}_{2 k} \mathrm{M}_{0}$ and $\mathrm{C}_{k}^{2} \leq \mathrm{C}_{2 k} \mathrm{C}_{0}$.

Remark 2.2. The conditions appearing in Lemmas 2.4 and 2.5 are closely related to the ones appearing when defining the Denjoy-Carleman classes (see for instance [16]).

\section{Preliminaries on the Volterra operator}

Given $g \in \mathcal{H}(\mathbf{C})$ we denote by $\mathcal{M}_{g}, \mathcal{D}$ and $\mathcal{I}$ the multiplication, differentiation and integration operators respectively, i.e. for $f \in \mathcal{H}(\mathbf{C})$ we have

$$
\mathcal{M}_{g}(f)(z)=g(z) f(z), \quad \mathcal{D} f(z)=f^{\prime}(z), \quad \mathcal{I} f(z)=\int_{0}^{z} f(\xi) d \xi
$$

Of course $\mathcal{I}(\mathcal{H}(\mathbf{C}))=\mathcal{H}_{0}(\mathbf{C}), I d_{\mathcal{H}(\mathbf{C})}=\mathcal{D} \mathcal{I}$ and $I d_{\mathcal{H}_{0}(\mathbf{C})}=\mathcal{I D}$ where $I d_{X}$ stands for the identity operator acting on $X$. We denote by $S$ and $S^{-1}$ the shift and backwards 
shift operators defined by

$$
S^{-1} f(z)=\frac{f(z)-f(0)}{z}=\sum_{n=0}^{\infty} a_{n+1} u_{n}, \quad S f(z)=z f(z)=\sum_{n=1}^{\infty} a_{n-1} u_{n},
$$

for each $f=\sum_{n=0}^{\infty} a_{n} u_{n} \in \mathcal{H}(\mathbf{C})$. Using the notation $P_{m}(f)$ for the Taylor polynomial of degree $m$ and $R_{m} f=f-P_{m-1}(f)$ for the remainder of degree $m$ we have

$$
S^{m} f(z)=z^{m} f(z)=\sum_{k=m}^{\infty} a_{k-m} z^{k}, \quad S^{-m} f(z)=\sum_{k=0}^{\infty} a_{k+m} z^{k}=\frac{R_{m} f(z)}{z^{m}} .
$$

This gives that $S^{m} S^{-m} f=R_{m} f$ and $S^{-m} S^{m} f=f$ for $m \in \mathbf{N}$.

Since $\mathcal{P} \subset \mathcal{F}_{p}^{\phi}(\mathbf{C})$ we have that $f \in \mathcal{F}_{p}^{\phi}(\mathbf{C})$ if and only if $R_{m} f \in \mathcal{F}_{p}^{\phi}(\mathbf{C})$ for any $m \in \mathbf{N}, 0<p \leq \infty$ and $\phi \in \mathcal{W}$. Note that $\left\|S^{m} f\right\|_{\mathcal{F}_{p}^{\phi}}=\|f\|_{\mathcal{F}_{p}^{\phi(m)}}$ for each $0<p \leq \infty$ where $\phi_{(m)}$ was defined by $e^{-\phi_{(m)}(r)}=r^{m} e^{-\phi(r)}$.

Lemma 3.1. Let $m \in \mathbf{N}, 1 \leq p \leq \infty$ and $\phi \in \mathcal{W}$. Then

$$
\left\|S^{-m} f\right\|_{\mathcal{F}_{p}^{\phi}(m)} \leq(m+1)\|f\|_{\mathcal{F}_{p}^{\phi}}
$$

for $f=\sum_{k=0}^{\infty} a_{k} u_{k} \in \mathcal{F}_{p}^{\phi}(\mathbf{C})$.

Proof. For each $k \in \mathbf{N} \cup\{0\}, r>0$ and $p \geq 1$ we have $\left|a_{k}\right| r^{k} \leq M_{1}(f, r) \leq$ $M_{p}(f, r)$. Thus

$$
\left|a_{k}\right| C_{k}(\phi, p) \leq(2 \pi)^{-1 / p}\|f\|_{\mathcal{F}_{p}^{\phi}}, \quad k \in \mathbf{N} \cup\{0\} .
$$

Therefore $\left\|P_{m-1}(f)\right\|_{\mathcal{F}_{p}^{\phi}} \leq m\|f\|_{\mathcal{F}_{p}^{\phi}},\left\|R_{m}(f)\right\|_{\mathcal{F}_{p}^{\phi}} \leq(m+1)\|f\|_{\mathcal{F}_{p}^{\phi}}$ and

$$
\left\|S^{-m}(f)\right\|_{\mathcal{F}_{p}^{\phi}(m)}=\left\|R_{m}(f)\right\|_{\mathcal{F}_{p}^{\phi}} \leq(m+1)\|f\|_{\mathcal{F}_{p}^{\phi}}
$$

This finishes the proof.

As mentioned in the introduction the Volterra operator with symbol $g$ is defined by the formula

$$
V_{g}(f)(z)=\mathcal{I M}_{\mathcal{D} g}(z)=z \int_{0}^{1} f(t z) g^{\prime}(t z) d t, \quad z \in \mathbf{C},
$$

for each $f \in \mathcal{H}(\mathbf{C})$.

Note that $V_{g}=0$ for any constant function $g$ and that also $V_{g}(f) \in \mathcal{H}_{0}(\mathbf{C})$ for any $f \in \mathcal{H}(\mathbf{C})$. We shall consider the following modification to avoid these restrictions. For each $f, g \in \mathcal{H}(\mathbf{C})$ we write

$$
\tilde{V}_{g}(f)(z)=\frac{1}{z} \int_{0}^{z} f(\xi) D g(\xi) d \xi, \quad z \in \mathbf{C},
$$

where $D=\mathcal{D} S$, that is $D f(z)=\sum_{n=0}^{\infty}(n+1) a_{n} z^{n}=z f^{\prime}(z)+f(z)$.

Denoting $I=S^{-1} \mathcal{I}$, we have for $f=\sum_{n=0}^{\infty} a_{n} u_{n}$ that

$$
I f(z)=\sum_{n=0}^{\infty} \frac{a_{n}}{(n+1)} z^{n}=\frac{1}{z} \int_{0}^{z} f(\xi) d \xi
$$

and we obtain that $\tilde{V}_{g}=I \mathcal{M}_{D g}$. In this way $\tilde{V}_{g}$ is well defined for $g \in \mathcal{H}(\mathbf{C})$ and takes values in $\mathcal{H}(\mathbf{C})$. Moreover, for each $f, g \in \mathcal{H}(\mathbf{C})$

$$
\tilde{V}_{g}(f)=S^{-1} V_{S g}(f), \quad V_{g}(f)=S \tilde{V}_{S^{-1} g}(f) .
$$


Since $V_{g}$ is continuous (in the topology of the uniform convergence on compact sets) from $\mathcal{H}(\mathbf{C})$ into $\mathcal{H}_{0}(\mathbf{C})$ and the map given by $g \rightarrow V_{g}$ is linear and continuous from $\mathcal{H}_{0}(\mathbf{C})$ into the space of continuous linear operators, using (27) similar results hold for $\tilde{V}_{g}$. Next result is immediate from the definitions.

Lemma 3.2. Let $0<p, q \leq \infty, \phi, \psi \in \mathcal{W}$ and $g \in \mathcal{H}(\mathbf{C})$. Then $V_{g}$ is bounded from $\mathcal{F}_{p}^{\phi}(\mathbf{C})$ into $\mathcal{F}_{q}^{\psi}(\mathbf{C})$ if and only if $\tilde{V}_{S^{-1} g}$ is bounded from $\mathcal{F}_{p}^{\phi}(\mathbf{C})$ into $\mathcal{F}_{q}^{\psi_{(1)}}(\mathbf{C})$.

Other expressions for the operators above are given as follows:

Lemma 3.3. Let $f, g \in \mathcal{H}(\mathbf{C})$ with $f=\sum_{m=0}^{\infty} b_{m} u_{m}$ and $g=\sum_{n=0}^{\infty} a_{n} u_{n}$. Then

$$
\begin{aligned}
& V_{g}(f)(z)=\sum_{j=1}^{\infty} \frac{1}{j}\left(\sum_{n+m=j} n a_{n} b_{m}\right) z^{j}, \\
& \tilde{V}_{g}(f)(z)=\sum_{j=0}^{\infty} \frac{1}{j+1}\left(\sum_{n+m=j}(n+1) a_{n} b_{m}\right) z^{j} .
\end{aligned}
$$

Proof. The proof is straightforward using

$$
\begin{aligned}
V_{g}(f)(z) & =\sum_{n=1}^{\infty} n a_{n} z^{n}\left(\int_{0}^{1} f(z s) s^{n-1} d s\right)=\sum_{n=1}^{\infty} n a_{n} z^{n}\left(\sum_{m=0}^{\infty} \frac{b_{m}}{n+m} z^{m}\right) \\
& =\sum_{j=1}^{\infty} \frac{1}{j}\left(\sum_{n+m=j} n a_{n} b_{m}\right) z^{j} .
\end{aligned}
$$

The other formula follows from (27).

Remark 3.1. From (28) and (29) we obtain for any $f, g \in \mathcal{H}(\mathbf{C})$ and $k \in \mathbf{N}$,

$$
\begin{gathered}
V_{g}\left(u_{0}\right)=g-g(0), \quad \tilde{V}_{g}\left(u_{0}\right)=g, \quad V_{u_{0}}(f)=0, \quad \tilde{V}_{u_{0}}(f)=I f, \\
V_{g}\left(u_{k}\right)=u_{k} \sum_{n=1}^{\infty} \frac{n a_{n}}{n+k} u_{n}, \quad \tilde{V}_{g}\left(u_{k}\right)=u_{k} \sum_{n=0}^{\infty} \frac{(n+1) a_{n}}{n+k+1} u_{n},
\end{gathered}
$$

and

$$
V_{u_{k}}(f)=k u_{k} \sum_{n=0}^{\infty} \frac{b_{n}}{n+k} u_{n}, \quad \tilde{V}_{u_{k}}(f)=(k+1) u_{k} \sum_{n=0}^{\infty} \frac{b_{n}}{n+k+1} u_{n} .
$$

Let us reformulate the boundedness of $\tilde{V}_{g}$ acting on $\mathcal{F}_{2}^{\phi}(\mathbf{C})$. Note that for each $f=\sum_{m=0}^{\infty} b_{m} u_{m}$ we can write

$$
\|f\|_{\mathcal{F}_{2}^{\phi}}=\frac{1}{\sqrt{2 \pi}}\left(\sum_{m=0}^{\infty}\left|b_{m}\right|^{2} C_{m}^{2}(\phi, 2)\right)^{1 / 2}
$$

Proposition 3.4. Let $\phi, \psi \in \mathcal{W}$ and $g \in \mathcal{H}(\mathbf{C})$ with $g=\sum_{n=0}^{\infty} a_{n} u_{n}$. Then $\tilde{V}_{g}$ maps $\mathcal{F}_{2}^{\phi}(\mathbf{C})$ into $\mathcal{F}_{2}^{\psi}(\mathbf{C})$ if and only if the matrix $A=(a(m, j))_{m, j=0}^{\infty}$ given by

$$
a(m, j)= \begin{cases}\frac{j-m+1}{j+1} a_{j-m} \frac{C_{j}(\psi, 2)}{C_{m}(\phi, 2)}, & m \leq j \\ 0, & 0 \leq j<m\end{cases}
$$

defines a bounded operator on $\ell^{2}(\mathbf{N} \cup\{0\})$. 
Proof. Using (32) and (29) we obtain

$$
\begin{aligned}
\left\|\tilde{V}_{g}(f)\right\|_{\mathcal{F}_{2}^{\psi}} & =\frac{1}{\sqrt{2 \pi}} \sup _{\left\|\left(\gamma_{j}\right)\right\|_{2}=1}\left|\sum_{j=0}^{\infty} \frac{1}{j+1}\left(\sum_{m=0}^{j}(j-m+1) a_{j-m} b_{m}\right) C_{j}(\psi, 2) \gamma_{j}\right| \\
& =\frac{1}{\sqrt{2 \pi}} \sup _{\left\|\left(\gamma_{j}\right)\right\|_{2}=1}\left|\sum_{m=0}^{\infty}\left(\sum_{j=m}^{\infty} \frac{j-m+1}{j+1} a_{j-m} \frac{C_{j}(\psi, 2)}{C_{m}(\phi, 2)} \gamma_{j}\right) C_{m}(\phi, 2) b_{m}\right| .
\end{aligned}
$$

Hence

This gives the result.

$$
\left\|\tilde{V}_{g}\right\|=\sup _{\left\|\left(\gamma_{j}\right)\right\|_{2}=1}\left(\sum_{m=0}^{\infty}\left|\sum_{j=0}^{\infty} a(m, j) \gamma_{j}\right|^{2}\right)^{1 / 2} .
$$

The analysis of $V_{g}$ for $g \in \mathcal{P}$ actually depends only on the integration operator. Let us denote by $V_{k}$ and $\tilde{V}_{k}$ the operators $V_{u_{k}}$ and $\tilde{V}_{u_{k}}$ for $k \in \mathbf{N} \cup\{0\}$. Hence from (31) we obtain

$$
V_{0}=0, \quad V_{1}=\mathcal{I}, \quad V_{k}=k \mathcal{I} S^{k-1}, \quad k \in \mathbf{N},
$$

and

$$
\tilde{V}_{0}=I, \quad \tilde{V}_{k}=(k+1) I S^{k}, \quad k \in \mathbf{N} .
$$

In particular $V_{k}=S \tilde{V}_{k-1}$ for $k \in \mathbf{N}$. A simple consequence of Proposition 3.4 gives the following particular case.

Corollary 3.5. Let $k \in \mathbf{N} \cup\{0\}$ and $\phi, \psi \in \mathcal{W}$. Then $\tilde{V}_{k}$ maps $\mathcal{F}_{2}^{\phi}(\mathbf{C})$ into $\mathcal{F}_{2}^{\psi}(\mathbf{C})$ if and only if

$$
\sup _{m \geq 0} \frac{C_{m+k}(\psi, 2)}{(m+k+1) C_{m}(\phi, 2)}<\infty .
$$

The following reformulations are elementary and left to the reader.

Lemma 3.6. Let $k \in \mathbf{N}, \phi, \psi \in \mathcal{W}$ and $0<p, q \leq \infty$. The following statements are equivalent:

(i) $V_{k}: \mathcal{F}_{p}^{\phi}(\mathbf{C}) \rightarrow \mathcal{F}_{q}^{\psi}(\mathbf{C})$ is bounded.

(ii) $\tilde{V}_{k-1}: \mathcal{F}_{p}^{\phi}(\mathbf{C}) \rightarrow \mathcal{F}_{q}^{\psi_{(1)}}(\mathbf{C})$ is bounded.

(iii) $\mathcal{I}: \mathcal{F}_{p}^{\phi_{(k-1)}}(\mathbf{C}) \rightarrow \mathcal{F}_{q}^{\psi}(\mathbf{C})$ is bounded.

(iv) $I: \mathcal{F}_{p}^{\phi_{(k-1)}}(\mathbf{C}) \rightarrow \mathcal{F}_{q}^{\psi_{(1)}}(\mathbf{C})$ is bounded.

\section{On necessary conditions for the boundedness}

Taking into account that $V_{g}\left(u_{0}\right)=g-g(0)$ the first condition for $V_{g}$ to map $\mathcal{F}_{p}^{\phi}(\mathbf{C})$ into $\mathcal{F}_{q}^{\psi}(\mathbf{C})$ is that $g \in \mathcal{F}_{q}^{\psi}(\mathbf{C})$. In particular we have the following trivial necessary condition.

Proposition 4.1. Let $0<p, q \leq \infty, \phi, \psi \in \mathcal{W}$ and $0 \neq g \in \mathcal{H}_{0}(\mathbf{C})$. If $V_{g}: \mathcal{F}_{p}^{\phi}(\mathbf{C}) \rightarrow \mathcal{F}_{q}^{\psi}(\mathbf{C})$ is bounded, then there exists a constant $A>0$ such that

$$
M_{\infty}(g, r) \leq A K_{\psi, q}(r), \quad r>0,
$$

where

$$
K_{\psi, q}(z)=\sum_{k=0}^{\infty} C_{k}(\psi, q)^{-1} z^{k}, \quad z \in \mathbf{C} .
$$


Proof. Using (24) for $V_{g}\left(u_{0}\right)=g(z)=\sum_{n=1}^{\infty} b_{n} z^{n}$ we obtain

$$
\begin{aligned}
M_{\infty}(g, r) & \leq \sum_{n=0}^{\infty}\left|a_{n}\right| r^{n} \leq(2 \pi)^{-1 / q} \sum_{n=0}^{\infty} C_{n}(\psi, q)^{-1}\left\|V_{g}\left(u_{0}\right)\right\|_{\mathcal{F}_{q}^{\psi}} r^{n} \\
& \leq(2 \pi)^{1 / p-1 / q}\left\|V_{g}\right\| C_{0}(\phi, p)\left(\sum_{n=0}^{\infty} C_{n}(\psi, q)^{-1} r^{n}\right) .
\end{aligned}
$$

This shows (35).

Let us find a necessary condition for the boundedness of $V_{g}$ from $\mathcal{F}_{p}^{\phi}(\mathbf{C})$ into $\mathcal{F}_{q}^{\psi}(\mathbf{C})$ in the case $\mathcal{F}_{q}^{\psi}(\mathbf{C}) \subseteq \mathcal{F}_{p}^{\phi}(\mathbf{C})$.

Proposition 4.2. Let $0<p, q \leq \infty, \phi, \psi \in \mathcal{W}$ such that $\mathcal{F}_{q}^{\psi}(\mathbf{C}) \subseteq \mathcal{F}_{p}^{\phi}(\mathbf{C})$ and $0 \neq g \in \mathcal{H}_{0}(\mathbf{C})$. If $V_{g}: \mathcal{F}_{p}^{\phi}(\mathbf{C}) \rightarrow \mathcal{F}_{q}^{\psi}(\mathbf{C})$ is bounded then there exists $A>0$ such that

$$
M_{\infty}(g, r) \leq A \phi(r), \quad r>0 .
$$

Proof. Let $A_{0}=\max \left\{1,\left\|u_{0}\right\|_{\mathcal{F}_{p}^{\phi}}\right\}$ and $C=\|\operatorname{Id}\|_{\mathcal{F}_{q}^{\psi}(\mathbf{C}) \rightarrow \mathcal{F}_{p}^{\phi}(\mathbf{C})}$. We observe that $V_{g}\left(u_{0}\right)=g \in \mathcal{F}_{q}^{\psi}(\mathbf{C})$. Hence $g \in \mathcal{F}_{p}^{\phi}(\mathbf{C})$ and $\|g\|_{\mathcal{F}_{p}^{\phi}} \leq C\|g\|_{\mathcal{F}_{q}^{\psi}} \leq C\left\|V_{g}\right\| A_{0}$. Since $V_{g}(g)=\frac{g^{2}}{2}$ we also obtain

$$
\left\|\frac{g^{2}}{2}\right\|_{\mathcal{F}_{p}^{\phi}} \leq C\left\|\frac{g^{2}}{2}\right\|_{\mathcal{F}_{q}^{\psi}} \leq C^{2}\left\|V_{g}\right\|^{2}\left\|u_{0}\right\|_{\mathcal{F}_{p}^{\phi}} \leq\left(C\left\|V_{g}\right\| A_{0}\right)^{2} .
$$

This allows to iterate the procedure to obtain $\frac{g^{n}}{n !} \in \mathcal{F}_{p}^{\phi}(\mathbf{C})$ and $\left\|\frac{g^{n}}{n !}\right\|_{\mathcal{F}_{p}^{\phi}} \leq\left(C\left\|V_{g}\right\| A_{0}\right)^{n}$.

Recall that $\mathcal{F}_{p}^{\phi}(\mathbf{C})$ is a $\tilde{p}$-Banach space for $\tilde{p}=\min \{p, 1\}$. Hence if $\sum_{n}\left\|f_{n}\right\|_{\mathcal{F}_{p}^{\phi}}^{\tilde{\tilde{p}}}<$ $\infty$ implies that $\sum_{n} f_{n} \in \mathcal{F}_{p}^{\phi}$. Therefore choosing $K>C\left\|V_{g}\right\| A_{0}$ we conclude that $\sum_{n=0}^{\infty} \frac{\beta_{n} g^{n}}{K^{n} n !} \in \mathcal{F}_{p}^{\phi}(\mathbf{C})$ for any sequence of complex numbers with $\sup _{n}\left|\beta_{n}\right| \leq 1$.

In particular, choosing $\beta_{n}=1$ for all $n \geq 0$ we obtain $e^{g / K} \in \mathcal{F}_{p}^{\phi}(\mathbf{C})$. Therefore $\int_{\mathbf{C}} e^{-p\left(\phi(|z|)-\frac{\Re g(z)}{K}\right)} d m(z)<\infty$ and $\sup _{z \in \mathbf{C}} e^{-\phi(|z|)+\frac{\Re g(z)}{K}}<\infty$ in the cases $p<\infty$ and $p=\infty$ respectively. In both cases one gets $\Re(g(z)) \leq K \phi(|z|)+C$. Selecting $\beta_{n}$ as $(-1)^{n}, i^{n}$ and $(-i)^{n}$ one concludes that $|g(z)| \leq A \phi(|z|)$ for some constant $A>0$ and the proof is complete.

A simple consequence of Proposition 4.2 is the following corollary.

Corollary 4.3. Let $0<p \leq \infty, \phi(r)=\varphi_{\alpha, \beta, \gamma}$ for some $\alpha, \beta>0, \gamma \in \mathbf{R}$ and $g \in \mathcal{H}_{0}(\mathbf{C})$.

(i) Case $0<\alpha<1: V_{g}: \mathcal{F}_{p}^{\phi}(\mathbf{C}) \rightarrow \mathcal{F}_{p}^{\phi}(\mathbf{C})$ is bounded if and only if $g=0$.

(ii) Case $\alpha \geq 1$ : If $V_{g}: \mathcal{F}_{p}^{\phi}(\mathbf{C}) \rightarrow \mathcal{F}_{p}^{\phi}(\mathbf{C})$ is bounded then $g \in \mathcal{P}$ and $1 \leq \operatorname{deg}(g) \leq$ $\alpha$.

Let us now show that boundedness of $V_{g}$ or $\tilde{V}_{g}$ between spaces $H_{v}^{\infty}(\mathbf{C})$ or $\mathcal{F}_{p}^{\phi}(\mathbf{C})$ forces certain a priori conditions on the weights.

Proposition 4.4. Let $0<p, q \leq \infty, \phi, \psi \in \mathcal{W}, g(z)=\sum_{n=0}^{\infty} a_{n} z^{n} \in \mathcal{H}(\mathbf{C})$ and define $\Lambda=\left\{n: a_{n} \neq 0\right\}$. If $\tilde{V}_{g}: \mathcal{F}_{p}^{\phi}(\mathbf{C}) \rightarrow \mathcal{F}_{q}^{\psi}(\mathbf{C})$ is bounded and $k \in \Lambda$, then $\tilde{V}_{k}: \mathcal{F}_{p}^{\phi}(\mathbf{C}) \rightarrow \mathcal{F}_{q}^{\psi}(\mathbf{C})$ is also bounded and $\left\|\tilde{V}_{k}\right\| \leq \frac{\left\|\tilde{V}_{g}\right\|}{\left|a_{k}\right|}$. In particular, $I: \mathcal{F}_{p}^{\phi}(\mathbf{C}) \rightarrow$ $\mathcal{F}_{q}^{\psi}(\mathbf{C})$ is bounded whenever $g(0) \neq 0$. 
Proof. Let $k \in \Lambda$. We have

$$
(k+1) a_{k} w^{k}=\int_{0}^{2 \pi} D g\left(e^{i \theta} w\right) e^{-i k \theta} \frac{d \theta}{2 \pi}, \quad w \in \mathbf{C},
$$

and therefore

$$
\begin{aligned}
\tilde{V}_{k} f(z) & =\frac{1}{z} \int_{0}^{z} f(w)(k+1) w^{k} d w=\frac{1}{a_{k}} \frac{1}{z} \int_{0}^{z} f(w)\left(\int_{0}^{2 \pi} D g\left(e^{i \theta} w\right) e^{-i k \theta} \frac{d \theta}{2 \pi}\right) d w \\
& =\frac{1}{a_{k}} \int_{0}^{2 \pi}\left(\frac{1}{z} \int_{0}^{z} f(w) D g\left(e^{i \theta} w\right) d w\right) e^{-i k \theta} \frac{d \theta}{2 \pi} .
\end{aligned}
$$

Hence, making the change of variable $e^{i \theta} w=w^{\prime}$ and denoting $f_{e^{-i \theta}}(z)=f\left(e^{-i \theta} z\right)$, we have

$$
\tilde{V}_{k} f(z)=\frac{1}{a_{k}} \int_{0}^{2 \pi} \tilde{V}_{g}\left(f_{e^{-i \theta}}\right)\left(e^{i \theta} z\right) e^{-i k \theta} \frac{d \theta}{2 \pi}
$$

In particular,

$$
\left\|\tilde{V}_{k} f\right\|_{\mathcal{F}_{q}^{\psi}} \leq \frac{1}{\left|a_{k}\right|} \int_{0}^{2 \pi}\left\|\tilde{V}_{g}\left(f_{e^{-i \theta}}\right)\left(e^{i \theta} z\right)\right\|_{\mathcal{F}_{q}^{\psi}} \frac{d \theta}{2 \pi}, \quad 1 \leq q \leq \infty
$$

and

$$
\left\|\tilde{V}_{k} f\right\|_{\mathcal{F}_{q}^{\psi}}^{q} \leq \frac{1}{\left|a_{k}\right|^{q}} \int_{0}^{2 \pi}\left\|\tilde{V}_{g}\left(f_{e^{-i \theta}}\right)\left(e^{i \theta} z\right)\right\|_{\mathcal{F}_{q}^{\psi}}^{q} \frac{d \theta}{2 \pi}, \quad 0<q<1 .
$$

This gives, taking into account that $\left\|f_{e^{-i \theta}}\right\|_{\mathcal{F}_{p}^{\phi}}=\|f\|_{\mathcal{F}_{p}^{\phi}}$ for any radial weight, the estimate $\left\|\tilde{V}_{k} f\right\|_{\mathcal{F}_{q}^{\psi}} \leq \frac{\left\|\tilde{V}_{g}\right\|}{\left|a_{k}\right|}\|f\|_{\mathcal{F}_{p}^{\phi}}$ and the proof is complete.

Corollary 4.5. Let $0<p, q \leq \infty, \phi, \psi \in \mathcal{W}$ and $0 \neq g \in \mathcal{H}(\mathbf{C})$. If $\tilde{V}_{g}: \mathcal{F}_{p}^{\phi}(\mathbf{C}) \rightarrow$ $\mathcal{F}_{q}^{\psi}(\mathbf{C})$ is bounded then there exists $k \in \mathbf{N}$ and $A_{k}>0$ such that

$$
C_{n+k}(\psi, q) \leq A_{k}(n+1) C_{n}(\phi, p), \quad n \geq 0 .
$$

In particular,

$$
I K_{\phi, p}(r) \leq A_{k} S^{-k} K_{\psi, q}(r), \quad r>0,
$$

where $K_{\phi, p}$ stands for the kernel given in (36).

Proof. Since $0 \neq g$ there exists $k \in \Lambda$, that is $a_{k} \neq 0$. Due to Proposition 4.4 and the fact $\tilde{V}_{k}\left(u_{n}\right)=(k+1) I\left(u_{n+k}\right)=\frac{k+1}{n+k+1} u_{n+k}$ we have $\frac{k+1}{n+k+1}\left\|u_{n+k}\right\|_{\mathcal{F}_{q}^{\psi}} \leq$ $\left\|\tilde{V}_{k}\right\|\left\|u_{n}\right\|_{\mathcal{F}_{q}^{\psi}}$. In particular, for all $n \in \mathbf{N}$,

$$
\left\|u_{n+k}\right\|_{\mathcal{F}_{q}^{\psi}} \leq\left\|\tilde{V}_{k}\right\|(n+1)\left\|u_{n}\right\|_{\mathcal{F}_{q}^{\psi}}
$$

This shows that

$$
I K_{\phi, p}(r)=\sum_{n=0}^{\infty} \frac{r^{n}}{(n+1) C_{n}(\phi, q)} \leq A_{k} \sum_{n=0}^{\infty} \frac{r^{n}}{C_{n+k}(\psi, q)}=A_{k} S^{-k} K_{\psi, q}(r),
$$

and the proof is complete.

Corollary 4.6. Let $0<p, q \leq \infty, \phi, \psi \in \mathcal{W}$ and let $g(z)=\sum_{n=1}^{\infty} a_{n} z^{n} \in \mathcal{H}_{0}(\mathbf{C})$ such that $V_{g}: \mathcal{F}_{p}^{\phi}(\mathbf{C}) \rightarrow \mathcal{F}_{q}^{\psi}(\mathbf{C})$ is bounded. Then $V_{k}: \mathcal{F}_{p}^{\phi}(\mathbf{C}) \rightarrow \mathcal{F}_{q}^{\psi}(\mathbf{C})$ is also bounded for each $k$ such that $g^{(k)}(0) \neq 0$. Moreover, the estimate $\left\|V_{k}\right\| \leq \frac{k !}{\left|g^{(k)}(0)\right|}\left\|V_{g}\right\|$ holds. In particular, $\mathcal{I}: \mathcal{F}_{p}^{\phi}(\mathbf{C}) \rightarrow \mathcal{F}_{q}^{\psi}(\mathbf{C})$ is bounded whenever $g^{\prime}(0) \neq 0$. 
Proof. Recall that due to Lemma 3.2 we have that $\tilde{V}_{S^{-1} g}: \mathcal{F}_{p}^{\phi}(\mathbf{C}) \rightarrow \mathcal{F}_{q}^{\psi_{(1)}}(\mathbf{C})$ where $e^{-\psi_{(1)}(r)}=r e^{-\psi(r)}$. Therefore invoking Proposition 4.4 and Lemma 3.6 we obtain that $V_{k}: \mathcal{F}_{p}^{\phi}(\mathbf{C}) \rightarrow \mathcal{F}_{q}^{\psi}(\mathbf{C})$ whenever $g^{(k)}(0) \neq 0$ and the corresponding estimate in norm holds.

Corollary 4.7. Let $\alpha_{i}, \beta_{i}>0$ and $\gamma_{i} \geq 0$ for $i=1,2, v(r)=e^{-\varphi_{\alpha_{1}, \beta_{1}, \gamma_{1}}(r)}$ and $w(r)=e^{-\varphi_{\alpha_{2}, \beta_{2}, \gamma_{2}}(r)}$ and $0 \neq g \in \mathcal{H}_{0}(\mathbf{C})$. Assume that $V_{g}: H_{v}^{\infty}(\mathbf{C}) \rightarrow H_{w}^{\infty}(\mathbf{C})$ is bounded. Then either $\alpha_{1}<\alpha_{2}$ or $\alpha_{1}=\alpha_{2}$ and $\beta_{1} \leq \beta_{2}$ or $\alpha_{1}=\alpha_{2}, \beta_{1}=\beta_{2}$ and $\gamma_{2} \leq \gamma_{1}+\alpha_{1}-1$. Moreover, in the case $\alpha_{1}=\alpha_{2}, \beta_{1}=\beta_{2}$ and $\delta=\alpha_{1}-\gamma_{2}+\gamma_{1} \geq 1$, then $g \in \mathcal{P}$ with $\operatorname{deg}(g) \leq \delta$.

Proof. Due to Corollary 4.6 we have that $V_{k}$ is bounded from $H_{v}^{\infty}(\mathbf{C})$ into $H_{w}^{\infty}(\mathbf{C})$ for all $k \in \mathbf{N}$ such that $g^{(k)}(0) \neq 0$. Since $V_{k}\left(u_{n}\right)=\frac{k}{n+k} u_{n+k}$ we have

$$
C_{n+k}\left(\varphi_{\alpha_{2}, \beta_{2}, \gamma_{2}}, \infty\right) \leq\left\|V_{k}\right\| \frac{n+k}{k} C_{n}\left(\varphi_{\alpha_{1}, \beta_{1}, \gamma_{1}}, \infty\right), \quad n \in \mathbf{N}
$$

Now take into account Example 2.1 to obtain for all $n \in \mathbf{N}$

$$
\left(\alpha_{2} \beta_{2}\right)^{-\frac{k+n+\gamma_{2}}{\alpha_{2}}}\left(k+n+\gamma_{2}\right)^{\frac{k+n+\gamma_{2}}{\alpha_{2}}} e^{-\frac{k+n+\gamma_{2}}{\alpha_{2}}} \leq\left\|V_{k}\right\| \frac{n+k}{k}\left(\alpha_{1} \beta_{1}\right)^{-\frac{n+\gamma_{1}}{\alpha_{1}}}\left(n+\gamma_{1}\right)^{\frac{n+\gamma_{1}}{\alpha_{1}}} e^{-\frac{n+\gamma_{1}}{\alpha_{1}}} .
$$

Hence there exists $C>0$ such that

$$
n^{n\left(\frac{1}{\alpha_{2}}-\frac{1}{\alpha_{1}}\right)} \leq C\left(\alpha_{2} \beta_{2} e\right)^{\frac{n}{\alpha_{2}}}\left(\alpha_{1} \beta_{1} e\right)^{-\frac{n}{\alpha_{1}}} n^{1-\frac{k+\gamma_{2}}{\alpha_{2}}+\frac{\gamma_{1}}{\alpha_{1}}}, \quad \forall n \in \mathbf{N} .
$$

This implies that $\alpha_{1} \leq \alpha_{2}$.

In the case $\alpha_{1}=\alpha_{2}$ the inequality becomes $\left(\frac{\beta_{1}}{\beta_{2}}\right)^{\frac{n}{\alpha_{1}}} \leq C n^{1-\frac{k+\gamma_{2}-\gamma_{1}}{\alpha_{1}}}$ for all $n \in \mathbf{N}$. This gives $\beta_{1} \leq \beta_{2}$.

Finally in the case $\alpha_{1}=\alpha_{2}, \beta_{1}=\beta_{2}$ we would have $n^{\frac{k+\gamma_{2}-\gamma_{1}}{\alpha_{1}}-1} \leq C$ for all $n \in \mathbf{N}$. This implies $\frac{k+\gamma_{2}-\gamma_{1}}{\alpha_{1}} \leq 1$. This gives, in particular, $\gamma_{2} \leq \gamma_{1}+\alpha_{1}-1$.

To finish the proof notice that $g^{(k)}(0) \neq 0$ implies $k \leq \alpha_{1}-\gamma_{2}+\gamma_{1}$ which implies that $g \in \mathcal{P}$ with $\operatorname{deg}(g) \leq \alpha_{1}-\gamma_{2}+\gamma_{1}$.

\section{On sufficient conditions for the boundedness}

Let us start presenting some sufficient conditions for the operators $V_{g}$ and $\tilde{V}_{g}$ to be bounded from $\mathcal{F}_{p}^{\phi}(\mathbf{C})$ into $\mathcal{F}_{p}^{\psi}(\mathbf{C})$ for any $1 \leq p \leq \infty$ and for general weights.

Proposition 5.1. Let $\phi, \psi \in \mathcal{W}$ and $g \in \mathcal{H}(\mathbf{C})$. Let us write $g_{r}(z)=g(r z)$ for $r>0$ and set

$$
A(\phi, \psi)=\sup _{r>0} e^{\phi(r)-\psi(r)}\left\|g_{r}\right\|_{B M O A}
$$

and

$$
B(\phi, \psi)=\sup _{r>0} e^{\phi(r)-\psi(r)}\left\|\left(g_{r}\right)^{\prime}\right\|_{H^{1}}
$$

(i) If $A(\phi, \psi)<\infty$, then both $\tilde{V}_{g}$ and $V_{g}$ are bounded from $\mathcal{F}_{p}^{\phi}(\mathbf{C})$ into $\mathcal{F}_{p}^{\psi}(\mathbf{C})$ for any $1 \leq p<\infty$.

(ii) If $B(\phi, \psi)<\infty$, then both $\tilde{V}_{g}$ and $V_{g}$ are bounded from $\mathcal{F}_{\infty}^{\phi}(\mathbf{C})$ into $\mathcal{F}_{\infty}^{\psi}(\mathbf{C})$. 
Proof. (i) Let $1 \leq p<\infty$ and set $A(\phi, \psi)=A$. Since $\left(g_{r}\right)^{\prime}(w)=r g^{\prime}(r w)$ for each $r>0$ and $|w|<1$, we have

$$
V_{g}(f)(r w)=\int_{0}^{w} f_{r}(\xi)\left(g_{r}\right)^{\prime}(\xi) d \xi
$$

Hence, using the estimate (1) we have

$$
M_{p}\left(V_{g}(f), r\right) \leq C_{p}\left\|g_{r}\right\|_{B M O A} M_{p}(f, r), \quad r>0 .
$$

Since $\tilde{V}_{g}=S^{-1} V_{S g},(S g)_{r}=r S\left(g_{r}\right)$ and $\left\|S g_{r}\right\|_{B M O A}=\left\|g_{r}\right\|_{B M O A}$, we also have

$$
M_{p}\left(\tilde{V}_{g}(f), r\right)=\frac{1}{r} M_{p}\left(V_{S g}(f), r\right) \leq C_{p}\left\|g_{r}\right\|_{B M O A} M_{p}(f, r) .
$$

Therefore, we conclude that

$$
\begin{aligned}
\max \left\{\left\|V_{g}(f)\right\|_{\mathcal{F}_{p}^{\psi}},\left\|\tilde{V}_{g}(f)\right\|_{\mathcal{F}_{p}^{\psi}}\right\} & \leq 2 \pi C_{p}^{p} \int_{0}^{\infty} M_{p}^{p}(f, r)\left\|g_{r}\right\|_{B M O A}^{p} r e^{-p \psi(r)} d r \\
& \leq 2 \pi C_{p}^{p} A^{p} \int_{0}^{\infty} M_{p}^{p}(f, r) r e^{-p \phi(r)} d r=C_{p}^{p} A^{p}\|f\|_{\mathcal{F}_{p}^{\phi}}^{p}
\end{aligned}
$$

(ii) Let $p=\infty$ and set $B(\phi, \psi)=B$. Without loss of generality we can assume that $g \in \mathcal{H}_{0}(\mathbf{C})$. Hence $g(z)=z \int_{0}^{1} g^{\prime}(z t) d t$ and thus $M_{1}(g, r) \leq r M_{1}\left(g^{\prime}, r\right)=$ $\left\|\left(g_{r}\right)^{\prime}\right\|_{H^{1}}$. In particular,

$$
M_{1}(D g, r) \leq r M_{1}\left(g^{\prime}, r\right)+M_{1}(g, r) \leq 2 r M_{1}\left(g^{\prime}, r\right)=2\left\|\left(g_{r}\right)^{\prime}\right\|_{H^{1}} .
$$

Hardy's inequality (see [14]) gives for $f(z)=\sum_{n=0}^{\infty} a_{n} z^{n}$

$$
M_{\infty}(I f, r) \leq \sum_{n=0}^{\infty} \frac{\left|a_{n}\right| r^{n}}{n+1} \leq C_{0} M_{1}(f, r), \quad r>0 .
$$

Therefore,

$$
\begin{aligned}
M_{\infty}\left(\tilde{V}_{g} f, r\right) & \leq C_{0} M_{1}((D g) f, r) \leq C_{0}\|f\|_{\mathcal{F}_{\infty}^{\phi}(\mathbf{C})} e^{\phi(r)} M_{1}(D g, r) \\
& \leq 2 B C_{0}\|f\|_{\mathcal{F}_{\infty}^{\phi}(\mathbf{C})} e^{\psi(r)} .
\end{aligned}
$$

This gives the boundedness of $\tilde{V}_{g}$ from $\mathcal{F}_{\infty}^{\phi}(\mathbf{C})$ into $\mathcal{F}_{\infty}^{\psi}(\mathbf{C})$.

To handle the case $V_{g}$ we use that $M_{1}\left(D\left(S^{-1} g\right), r\right)=M_{1}\left(g^{\prime}, r\right)$. Arguing as above, we have

$$
M_{\infty}\left(V_{g} f, r\right)=r M_{\infty}\left(\tilde{V}_{S^{-1} g} f, r\right) \leq C\|f\|_{\mathcal{F}_{\infty}^{\phi}(\mathbf{C})} e^{\phi(r)}\left\|\left(g_{r}\right)^{\prime}\right\|_{H^{1}}
$$

and the result follows with the same argument.

Proposition 5.2. Let $\phi, \psi \in \mathcal{W}$ where $\psi$ is differentiable with $\psi^{\prime}(t)>0$ for $t>0$ and $g \in \mathcal{H}_{0}(\mathbf{C})$. Set

$$
B_{1}(\phi, \psi)=\sup _{r>0} \frac{e^{\phi(r)-\psi(r)}}{r \psi^{\prime}(r)}\left\|\left(g_{r}\right)^{\prime}\right\|_{H^{\infty}} .
$$

If $B_{1}(\phi, \psi)<\infty$, then both $\tilde{V}_{g}$ and $V_{g}$ are bounded from $H_{v}^{\infty}(\mathbf{C})$ to $H_{w}^{\infty}(\mathbf{C})$, where $v(z)=e^{-\phi(|z|)}$ and $w(z)=e^{-\psi(|z|)}$. 
Proof. Let $B_{1}(\phi, \psi)=B_{1}$. Arguing as in (41) we obtain that $M_{\infty}(D g, r) \leq$ $2\left\|\left(g_{r}\right)^{\prime}\right\|_{H^{\infty}}$. Now for $|z|=r$ we can estimate

$$
\begin{aligned}
\left|\tilde{V}_{g}(f)(z)\right| & \leq \int_{0}^{1}|f(z t)||D g(z t)| d t \leq \int_{0}^{1} M_{\infty}(f, r t) M_{\infty}(D g, r t) d t \\
& \leq \frac{1}{r} \int_{0}^{r} M_{\infty}(f, t) M_{\infty}(D g, t) d t=2\|f\|_{v} \frac{1}{r} \int_{0}^{r} e^{\phi(t)}\left\|\left(g_{t}\right)^{\prime}\right\|_{H^{\infty}} d s \\
& \leq 2 B_{1}\|f\|_{v} \frac{1}{r} \int_{0}^{r} t \psi^{\prime}(t) e^{\psi(t)} d s=2 B_{1}\|f\|_{v}\left(e^{\psi(r)}-e^{\psi(0)}\right) \leq 2 B_{1}\|f\|_{v} e^{\psi(|z|)} .
\end{aligned}
$$

This completes the proof for $\tilde{V}_{g}$. The case $V_{g}$ follows similarly using that

$$
M_{\infty}\left(D\left(S^{-1} g\right), r\right)=M_{\infty}\left(g^{\prime}, r\right) .
$$

Let us apply the previous result to polynomials, in particular for $V_{k}=V_{u_{k}}$.

Corollary 5.3. Let $v(z)=e^{-\varphi_{\alpha, \beta, \gamma}}$ for some $\beta>0, \gamma \in \mathbf{R}$ and $\alpha \geq 1$ and let $g \in \mathcal{H}_{0}(\mathbf{C})$. Then the following statements are equivalent:

(i) $V_{g}: H_{v}^{\infty}(\mathbf{C}) \rightarrow H_{v}^{\infty}(\mathbf{C})$ is bounded.

(ii) $g \in \mathcal{P}$ and $1 \leq \operatorname{deg}(g) \leq[p]$.

Proof. (i) $\Longrightarrow$ (ii) This is the case $p=\infty$ in Corollary 4.3.

(ii) $\Longrightarrow$ (i). It suffices to show that $V_{k}$ is bounded on $H_{v}^{\infty}(\mathbf{C})$ for $1 \leq k \leq \alpha$. Now for each $1 \leq k \leq[\alpha]$ we have

$$
\lim _{r \rightarrow \infty} \frac{\varphi_{\alpha, \beta, \gamma}^{\prime}(r)}{r^{k-1}}= \begin{cases}\alpha \beta, & k=\alpha ; \\ \infty, & k<\alpha .\end{cases}
$$

We can then apply Proposition 5.2 for $\phi=\psi=\varphi_{\alpha, \beta, \gamma}$ and $g=u_{k}$ to finish the proof.

Let us get now some conditions depending on $p$ for the boundedness on $\mathcal{F}_{p}^{\phi}(\mathbf{C})$. We shall use the following result.

Lemma 5.4. Let $0<p<\infty, \phi \in \mathcal{W}$. If $f \in \mathcal{F}_{p}^{\Phi_{p}}(\mathbf{C})$, then $I(f) \in \mathcal{F}_{p}^{\phi}(\mathbf{C})$.

Proof. Using that $\operatorname{If}(z)=\int_{0}^{1} f(z t) d t$, for any $0<p<\infty$ we obtain

$$
M_{p}^{p}(I(f), r) \leq \int_{0}^{1} M_{p}^{p}(f, r t) d t \leq \frac{1}{r} \int_{0}^{r} M_{p}^{p}(f, t) d t
$$

Therefore,

$$
\begin{aligned}
\|I(f)\|_{\mathcal{F}_{p}^{\phi}}^{p} & \leq C \int_{0}^{\infty}\left(\int_{0}^{r} M_{p}^{p}(f, t) d t\right) e^{-p \phi(r)} d r \leq C \int_{0}^{\infty} M_{p}^{p}(f, t)\left(\int_{t}^{\infty} e^{-p \phi(r)} d r\right) d t \\
& \leq C \int_{0}^{\infty} M_{p}^{p}(f, t) t e^{-p \Phi_{p}(t)} d t=C\|f\|_{\mathcal{F}_{p}^{\Phi_{p}}}^{p} .
\end{aligned}
$$

The proof is now complete.

Proposition 5.5. Let $0<p<\infty, \phi, \psi \in \mathcal{W}$ and set

$$
A_{1}(\phi, \psi, p)=\sup _{r>0} e^{\phi(r)-\Psi_{p}(r)} M_{\infty}(D g, r) .
$$

If $A_{1}(\phi, \psi, p)<\infty$, then $\tilde{V}_{g}$ is bounded from $\mathcal{F}_{p}^{\phi}(\mathbf{C})$ into $\mathcal{F}_{p}^{\psi}(\mathbf{C})$. 
Proof. Let $A_{1}(\phi, \psi, p)=A_{1}$. Using Lemma 5.4 and recalling that $r e^{-p \Psi_{p}(r)}=$ $\int_{r}^{\infty} e^{-p \psi(s)} d s$ we have

$$
\begin{aligned}
\left\|\tilde{V}_{g}(f)\right\|_{\mathcal{F}_{p}^{\psi}}^{p} & =\left\|I M_{D g} f\right\|_{\mathcal{F}_{p}^{\psi}}^{p} \leq C\left\|M_{D g} f\right\|_{\mathcal{F}_{p}^{\Psi p}}^{p} \\
& \leq C \int_{0}^{\infty} M_{p}^{p}(f, r) M_{\infty}^{p}(D g, r)\left(\int_{r}^{\infty} e^{-p \psi(s)} d s\right) d r \\
& \leq C A_{1}^{p} \int_{0}^{\infty} M_{p}^{p}(f, r) r e^{-p \phi(r)} d r
\end{aligned}
$$

The proof is finished.

We can actually weaken the condition (43) in the case $p>1$ using the following modification of the $p$-distortion functions.

Definition 5.1. Let $\psi, \phi \in \mathcal{W}$ and $0<p<\infty$. We define

$$
H_{\psi, \phi, p}(r)=e^{\Psi_{p}(r)-\phi(r)}, \quad 0<p \leq 1,
$$

and

$$
H_{\psi, \phi, p}(r)=\frac{r e^{-(\phi(r)+(p-1) \psi(r))}}{\int_{r}^{\infty} e^{-p \psi(s)} d s}, \quad 1<p<\infty .
$$

In particular, $H_{\phi, \phi, p}(r)=e^{\max \{p, 1\}\left(\Phi_{p}(r)-\phi(r)\right)}$.

Remark 5.1. Note that for $p \geq 1$ we can write

$$
H_{\psi, \phi, p}(r)=e^{\Psi_{p}(r)-\phi(r)} e^{(p-1)\left(\Psi_{p}(r)-\psi(r)\right)}=e^{p\left(\Psi_{p}(r)-\psi(r)\right)} e^{\psi(r)-\phi(r)} .
$$

In particular, due to (ii) in Lemma 2.1 if $\psi$ is differentiable and convex, then

$$
e^{\Psi_{p}(r)-\phi(r)} \leq C H_{\psi, \phi, p}(r), \quad r>R,
$$

and for $\psi \in \mathcal{W}_{0}$, from Proposition 2.3, one has

$$
e^{\psi(r)-\phi(r)} \leq C H_{\psi, \phi, p}(r), \quad r>R .
$$

We shall use the following general fact.

Lemma 5.6. Let $1 \leq p<\infty$, let $U, W:(0, \infty) \rightarrow(0, \infty)$ be measurable functions with $W \in L^{1}((0, \infty))$ and let $G:[0, \infty) \rightarrow \mathbf{R}^{+}$be a continuous function. Assume that there exists $C>0$ such that

$$
G(r) \leq C\left(\frac{1}{r} \int_{r}^{\infty} W(t) d t\right)^{-1} U^{1 / p}(r) W^{1 / p^{\prime}}(r), \quad r>0 .
$$

Then

$$
\int_{0}^{\infty}\left(\frac{1}{r} \int_{0}^{r} F(t) G(t) d t\right)^{p} r W(r) d r \leq C \int_{0}^{\infty} F^{p}(r) r U(r) d r
$$

for any continuous function $F:[0, \infty) \rightarrow \mathbf{R}^{+}$.

Proof. For $p=1$ condition (45) becomes $G(t)\left(\int_{t}^{\infty} W(r) d r\right) \leq C t U(t)$ for $t>0$ and the result follows from Fubini's theorem.

Assume $p>1$. For each $R, \varepsilon>0$ integrating by parts we have

$$
\int_{\varepsilon}^{R}\left(\frac{1}{r} \int_{0}^{r} F(t) G(t) d t\right)^{p} r W(r) d r
$$




$$
\begin{aligned}
= & \left(\int_{0}^{\varepsilon} F(t) G(t) d t\right)^{p}\left(\int_{\varepsilon}^{\infty} \frac{W(t)}{t^{p-1}} d t\right)-\left(\int_{0}^{R} F(t) G(t) d t\right)^{p}\left(\int_{R}^{\infty} \frac{W(t)}{t^{p-1}} d t\right) \\
& +p \int_{0}^{R}\left(\int_{0}^{r} F(t) G(t) d t\right)^{p-1} F(r) G(r)\left(\int_{r}^{\infty} \frac{W(t)}{t^{p-1}} d t\right) d r \\
\leq & \left(\varepsilon \int_{0}^{\infty} W(t) d t\right)\left(\frac{1}{\varepsilon} \int_{0}^{\varepsilon} F(t) G(t) d t\right)^{p} \\
& +p \int_{0}^{\infty}\left(\int_{0}^{r} F(t) G(t) d t\right)^{p-1} F(r) G(r)\left(\int_{r}^{\infty} \frac{W(t)}{t^{p-1}} d t\right) d r .
\end{aligned}
$$

Now passing to the limit as $R \rightarrow \infty$ and $\varepsilon \rightarrow 0$, applying (45) and Hölder's inequality we have

$$
\begin{aligned}
& \int_{0}^{\infty}\left(\frac{1}{r} \int_{0}^{r} F(t) G(t) d t\right)^{p} r W(r) d r \int_{0}^{\infty}\left(\int_{0}^{r} F(t) G(t) d t\right)^{p-1} F(r) G(r)\left(\int_{r}^{\infty} \frac{W(t)}{t^{p-1}} d t\right) d r \\
& \leq p \int_{0}^{\infty}\left(\frac{1}{r} \int_{0}^{r} F(t) G(t) d t\right)^{p-1} F(r) G(r)\left(\int_{r}^{\infty} W(t) d t\right) d r \\
& \leq C\left(\int_{0}^{\infty}\left(\frac{1}{r} \int_{0}^{r} F(t) G(t) d t\right)^{p} r W(r) d r\right)^{1 / p^{\prime}} \\
& \quad \cdot\left(\int_{0}^{\infty} F(r)^{p} G(r)^{p} W^{1-p}(r) r\left(\frac{1}{r} \int_{r}^{\infty} W(t) d t\right)^{p} d r\right)^{1 / p} \\
& \leq C\left(\int_{0}^{\infty}\left(\frac{1}{r} \int_{0}^{r} F(t) G(t) d t\right)^{p} r W(r) d r\right)^{1 / p^{\prime}}\left(\int_{0}^{\infty} F^{p}(r) r U(r) d r\right)^{1 / p} .
\end{aligned}
$$

This implies (46) and the proof is then complete.

Theorem 5.7. Let $0<p<\infty, \phi, \psi \in \mathcal{W}$ and $g \in \mathcal{H}(\mathbf{C})$. If there exists $A>0$ such that

$$
M_{\infty}(D g, r) \leq A H_{\psi, \phi, p}(r), \quad r>0
$$

then $\tilde{V}_{g}$ is bounded from $\mathcal{F}_{p}^{\phi}(\mathbf{C})$ into $\mathcal{F}_{p}^{\psi}(\mathbf{C})$.

Proof. The case $0<p \leq 1$ was shown in Proposition 5.5.

Let us assume now that $1<p<\infty$. Writing $\tilde{V}_{g}(f)(z)=\int_{0}^{1} f(z t) D g(z t) d t$ we have for $0<r<\infty$ and $\theta \in[0,2 \pi)$,

$$
\left|\tilde{V}_{g}(f)\left(r e^{i \theta}\right)\right| \leq \int_{0}^{1}\left|f\left(r e^{i \theta} t\right)\right| M_{\infty}(D g, r t) d t .
$$

Using vector-valued Minkowski's inequality we have

$$
M_{p}\left(\tilde{V}_{g}(f), r\right) \leq \frac{1}{r} \int_{0}^{r} M_{p}(f, t) M_{\infty}(D g, t) d t .
$$

Let $U(r)=e^{-p \phi(r)}$ and $W(r)=e^{-p \psi(r)}$ and observe that

$$
H_{\psi, \phi, p}(r)=\left(\frac{1}{r} \int_{r}^{\infty} W(t) d t\right)^{-1} U^{1 / p}(r) W^{1 / p^{\prime}}(r) .
$$


Consider now $F(t)=M_{p}(f, t)$ and $G(t)=M_{\infty}(D g, t)$ and notice that (47) together with (48) allow us to apply Lemma 5.6 to obtain

$$
\int_{0}^{\infty} M_{p}^{p}\left(\tilde{V}_{g}(f), r\right) r e^{-p \psi(r)} d r \leq C \int_{0}^{\infty} M_{p}^{p}(f, r) r e^{-p \phi(r)} d r .
$$

This finishes the proof.

We can now extend the condition in Proposition 5.2 also for boundedness in Fock-type spaces, at least for convex functions $\psi$.

Corollary 5.8. Let $0<p<\infty$ and let $\psi \in \mathcal{W}$ be differentiable and convex in $(0, \infty)$. If $g \in \mathcal{H}(\mathbf{C})$ satisfies

$$
\sup _{r>0} \frac{e^{\phi(r)-\psi(r)} M_{\infty}(D g, r)}{r \psi^{\prime}(r)}=A<\infty,
$$

then $\tilde{V}_{g}: \mathcal{F}_{p}^{\phi}(\mathbf{C}) \rightarrow \mathcal{F}_{p}^{\psi}(\mathbf{C})$ is bounded.

Proof. First observe that

$$
r \psi^{\prime}(r) e^{-p \Psi_{p}(r)} \leq \int_{r}^{\infty} \psi^{\prime}(s) e^{-p \psi(s)} d s \leq \frac{1}{p} e^{-p \psi(r)}, \quad r>0 .
$$

Hence assumption (49) gives

$$
M_{\infty}(D g, r) \leq \frac{A}{p} e^{(1-p)\left(\psi(r)-\Psi_{p}(r)\right)} e^{\Psi_{p}(r)-\phi(r)} .
$$

Hence, according to (44) we obtain the condition (47) in the case $p \geq 1$. On the other hand, for $0<p \leq 1$ due to part (ii) in Lemma 2.1 to know that $\sup _{r>0} e^{\psi(r)-\Psi_{p}(r)}<$ $\infty$. Hence $M_{\infty}(D g, r) \leq K e^{\Psi_{p}(r)-\phi(r)}=K H_{\psi, \phi, p}(r)$. The result now follows from Theorem 5.7.

Corollary 5.9. Let $0<p<\infty, \phi(r)=\varphi_{\alpha, \beta, \gamma}(r)$ for $\beta>0, \gamma \geq 0$ and $\alpha \geq 1$ and let $g \in \mathcal{H}_{0}(\mathbf{C})$. Then the following statements are equivalent:

(i) $V_{g}: \mathcal{F}_{p}^{\phi}(\mathbf{C}) \rightarrow \mathcal{F}_{p}^{\phi}(\mathbf{C})$ is bounded.

(ii) $g \in \mathcal{P}$ and $1 \leq \operatorname{deg}(g) \leq[\alpha]$.

Proof. (i) $\Longrightarrow$ (ii) This was shown in Corollary 4.3 .

(ii) $\Longrightarrow$ (i). Let $1 \leq k \leq[\alpha]$ and let us show that $V_{k}: \mathcal{F}_{p}^{\phi}(\mathbf{C}) \rightarrow \mathcal{F}_{p}^{\phi}(\mathbf{C})$ is bounded, or equivalently $\tilde{V}_{k-1}: \mathcal{F}_{p}^{\phi}(\mathbf{C}) \rightarrow \mathcal{F}_{p}^{\phi(1)}(\mathbf{C})$ is bounded. From Proposition 5.7 it suffices to see that (47) holds for $g(z)=z^{k-1}$. Recall that $H_{\psi, \phi, p}^{-1}(r)=$ $e^{p\left(\psi(r)-\Psi_{p}(r)\right)} e^{\phi(r)-\psi(r)}$ for $p \geq 1$ and $H_{\psi, \phi, p}^{-1}(r)=e^{\phi(r)-\Psi_{p}(r)}$ for $0<p<1$. Hence, in particular for $\psi=\phi_{(1)}=\varphi_{\alpha, \beta, \gamma+1}$ we have $\phi(r)-\psi(r)=\log (r)$, we obtain, invoking (iii) in Lemma 2.1, that

$$
H_{\psi, \phi, p}^{-1}(r) \leq C r^{-\alpha+1}, \quad r>0
$$

This gives

$$
\sup _{r \geq 1} H_{\psi, \phi, p}^{-1}(r) M_{\infty}\left(D u_{k-1}, r\right) \leq C_{k} \sup _{r \geq 1} r^{k-\alpha}<\infty .
$$

The proof is now complete.

Acknowledgements. I wish to thank J. Bonet for his comments on the first draft of the paper. I am also grateful to the referee for his/her careful reading. 


\section{References}

[1] Aleman, A.: A class of integral operators on spaces of analytic functions. - Topics in complex analysis and operator theory 330, Univ. Malaga, Malaga, 2007.

[2] Aleman, A., and O. Constantin: Spectra of integration operators on weighted Bergman spaces. - J. Anal. Math. 109, 2009, 199-231.

[3] Aleman, A., and J. A. PelÁez: Spectra of integration operators and weighted square functions. - Indiana Univ. Math. J. 61, 2012, 1-19.

[4] Aleman, A., and A. G. Siskakis: An integral operator on $H^{p}$. - Complex Var. Theory Appl. 28, 1995, 149-158.

[5] Aleman, A., and A. G. Siskakis: Integration operators on Bergman spaces. - Indiana Univ. Math. J. 46, 1997, 337-356.

[6] Basallote, M., M. D. Contreras, C. Hernández-Mancera, M. J. Martín, and P. J. PAÚl: Volterra operators and semigroups in weighted Banach spaces of analytic functions. Collect. Math. 65, 2014, 233-249.

[7] Blasco, O., and A. Galbis: On Taylor coefficient of entire functions integrable against exponential weights. - Math. Nachr. 223, 2001, 5-21.

[8] Bonet, J.: The spectrum of Volterra operators on weighted spaces of entire functions. - Quart. J. Math. 66, 2015, 799-807.

[9] Bonet, J., and J. TASkinen: A note on Volterra operators on weighted Banach spaces of entire functions. - Math. Nachr. 288:11-12, 2015, 1216-1225.

[10] Bierstedt, K. D., J. Bonet, and J. Taskinen: Associated weights and spaces of holomorphic functions. - Studia Math. 127, 1998, 137-168.

[11] Constantin, O.: A Volterra-type integration operator on Fock spaces. - Proc. Amer. Math. Soc. 140:12, 2012, 4247-4257.

[12] Constantin, O., and J.A. PelÁez: Integral operators, embedding theorems and a Littlewood-Paley formula on weighted Fock spaces. - J. Geom. Anal. 26:2, 2016, 1109-1154.

[13] Constantin, O., and A. M. Persson: The spectrum of Volterra-type integration operators on generalized Fock spaces. - Bull. London Math. Soc. 47:6, 2015, 958-963.

[14] Duren, P.: Theory of Hardy spaces. - Academic Press, New York-London, 1970.

[15] Hu, Z.: Extended Cesaro operators on mixed-norm spaces. - Proc. Amer. Math. Soc. 131:7, 2003, 2171-2179.

[16] Komatsu, H.: Ultradistributions I, structure theorems and a characterization. - J. Fac. Sci. Tokyo (IA) 20, 1973, 25-105.

[17] PAU, J., and J. A. PELÁEZ: Embedding theorems and integration operators on Bergman spaces with rapidly decreasing weights. - J. Funct. Anal. 259, 2010, 2727-2756.

[18] Pavlović, M. and J. A. Peláez: An equivalence for weighted integrals of an analytic function and its derivative. - Math. Nachr. 281:11, 2008, 1612-1623.

[19] Peláez, J.A., and J. RÄтtyä: Weighted Bergman Spaces induced by rapidly decreasing weights. - Mem. Amer. Math. Soc. 227, 2014.

[20] Pommerenke, Ch.: Schlichte Funktionen un analytische Functionen von beschränkter mittlerer Oszilation. - Comment. Math. Helv. 52, 1977, 591-602.

[21] Tung, J.: Fock spaces. - PhD dissertation, University of Michigan, 2005.

[22] ZHU, K.: Operator theory in function spaces. Second Edition. - Math. Surveys Monogr. 138, Amer. Math. Soc. Providence, Rhode Island, 2007.

[23] ZHu, K.: Analysis on Fock spaces. - Springer-Verlag, New York, 2012. 